\title{
Estudio experimental sobre estrategias didácticas innovadoras y tradicionales en la enseñanza de Estudios Sociales
}

\author{
Experimental study on innovative and traditional didactic strategies \\ in the teaching of Social Studies
}

Adolfo Alejandro Díaz Pérez ${ }^{1}$

\section{Resumen}

En los distintos espacios educativos es común hacer referencia a las metodologías innovadoras y a las metodologías tradicionales, máxime en el contexto actual en donde el flamante magisterio está siendo portavoz de cambios en las metodologías didácticas que desde antaño han estado bien enraizadas, particularmente, en la enseñanza de las ciencias sociales. Sin embargo, mientras la lucha entre lo innovador y lo tradicional se agudiza en los espacios de debates educativos, las prácticas pedagógicas tradicionales en el aula de clase permanecen inalterables y, por otra parte, los estudiantes -ahora al margen de los nuevos recursos que brinda la sociedad- aparcan expectantes por otras formas de aprender. A partir de esto, la presente investigación se propuso indagar la opinión del estudiantado acerca de las estrategias didácticas innovadoras y tradicionales, para ello se utilizó un diseño experimental exploratorio y se realizó una intervención didáctica de diez sesiones de clase, a fin de generar un espacio de reflexión entre el profesorado respecto a los retos de la innovación educativa.

Palabras clave: Aprendizaje de las ciencias sociales, estrategias didácticas, innovación, tradicionalismo.

\section{Abstract}

In the different educational spaces it is common to refer to innovative methodologies and traditional methodologies, especially in the current context where the new teaching is being spokesman of changes in teaching methodologies that have long been well rooted, particularly in teaching of the social sciences. However, while the struggle between the innovative and the traditional deepens in the spaces of educational debates, the traditional pedagogical practices in the classroom remain unchanged and, on the other hand, the students -now regardless of the new resources offered society- they are waiting expectantly for other ways of learning. From this, the

1 Licenciado en Ciencias de la Educación con mención en Ciencias Sociales. Profesor de la Facultad de Educación e Idiomas de la Universidad Nacional Autónoma de Nicaragua-Managua. Email: adolfoalejandro73@yahoo.com (D): https://orcid.org/o0oo-0002-4295-4094

Recibido: 02/02/2019 Aprobado: 05/05/2019 
present investigation was proposed to investigate the opinion of the student about the innovative and traditional didactic strategies, for this an exploratory experimental design was used and a didactic intervention of ten class sessions was carried out, in order to generate a space of reflection among teachers regarding the challenges of educational innovation.

Key Words: Learning of the social sciences, didactic strategies, innovation, traditionalism.

\section{Introducción}

La presente investigación es el resultado de un experimento didáctico llevado a cabo en el Colegio Público de Santo Domingo, Managua, con estudiantes de 4to grado de Educación Primaria en la asignatura Estudios Sociales. La investigación surgió de la inquietud de conocer la opinión que tiene el estudiantado respecto a las metodologías innovadoras y tradicionales en la enseñanza de los estudios sociales, ya que actualmente el aula de clase es escenario de una pugna constante entre ambos tipos de metodologías, y mientras las innovaciones educativas intentan posicionarse del escenario educativo, las metodologías tradicionales se niegan a dejar los espacios que desde antaño han ocupado.

Es así que, adherido a un diseño experimental aplicado a los procesos educativos, el docente investigador llevó a cabo una intervención didáctica con metodologías innovadoras y metodologías tradicionales, a fin de conocer cuál es la opinión que sostiene el estudiantado respecto a este tipo de metodologías. En este sentido, los resultados del estudio invitan al profesorado a reflexionar acerca de las tendencias actuales de la educación y de las expectativas que tiene el estudiantado de los procesos de aprendizajes. No obstante, en el presente escrito se describe el proceso de intervención didáctica, las actividades que llevaron a cabo los estudiantes, la opinión del estudiantado y los retos que ha de afrontar el profesorado de ciencias sociales en la presente coyuntura científica, tecnológica y social en la que se sitúan la educación.

\section{Literatura}

\section{Hacia una aproximación de lo tradicional y lo innovador}

En el ámbito de la educación es común que administradores de la educación, diseñadores curriculares, expertos en educación, asesores pedagógicos, directores, profesorado, inclusive, que el mismo estudiantado y la comunidad de padres de familia, hagan referencia a dos términos que son parte del tesauro propio de la educación: lo tradicional y lo innovador. Cuesta precisar con claridad cuáles son los límites del uno y del otro, y resulta hasta polémico calificar una práctica educativa como tradicional 
o innovadora, pues para cada uno existe un contexto particular, diversos criterios y una abundante bibliografía que lo definen de una manera particular.

Lo cierto es que hay un punto de partida que demarca continuamente la relación entre lo tradicional y lo innovador, esto son los constantes avances, cambios y transformaciones que experimenta la sociedad. Estos avances de la sociedad en materia de conocimiento, ciencia y tecnología exigen de la educación la incorporación de sucesivas innovaciones para hacer frente a los nuevos contextos que van surgiendo, porque como expresa López (2007, p.31) "la innovación debería ir siempre unida al concepto de cambio", lo cual, indudablemente, es vital para que la educación ocupe una posición frontal con la sociedad, y así competir efectivamente con aquel longevo adagio escolar que aún muestra el rezago de la gestión escolar: "tenemos escuelas del siglo XIX, maestros del XX y estudiantes del siglo XXI".

En otras palabras, existe una relación dialéctica entre lo tradicional y lo innovador, lo tradicional es el punto de origen de las corrientes innovadoras, o bien, junto a la permanencia de prácticas tradicionales que no se ajustan a las nuevas condiciones socioeducativas, van abriendo pasos otras prácticas innovadoras como alternativas remediales. Tal es el caso de las reformas educativas que se gestan continuamente en los sistemas educativos, por citar un ejemplo, el estudio de Santiago (2008) demuestra que las nuevas propuestas curriculares realizadas a partir de 1999 en la República Bolivariana de Venezuela, apuntan a incentivar a nuevas prácticas educativas con el objetivo de "formar ciudadanos conscientes de la complejidad de su mundo y de su entorno inmediato" (p.10), y superar asíla "orientación pedagógica tradicional" (p.16).

Y aunque es complejo precisar los límites entre lo tradicional y lo innovador porque es algo relativo que obedece a la realidad de cada contexto educativo-, existen ciertos indicadores que dan luces sobre qué puede considerarse una práctica tradicional o innovadora. En cuanto a las primeras, Rodríguez (2013) las describe muy ampliamente: "el método de enseñanza es eminentemente expositivo, la evaluación del aprendizaje es reproductiva, centrada en la calificación del resultado, la relación profesor-alumno es autoritaria, se fundamenta en la concepción del alumno como receptor de información" (p.39). De igual manera, Santiago (2008) argumenta que en las prácticas tradicionales "la actividad pedagógica es predominantemente dictar, copiar, dibujar y calcar y la evaluación es sumativa” (p.6).

En este sentido, este tipo de prácticas tradicionales en la enseñanza de las ciencias sociales es la que conllevan al profesorado a utilizar el mismo bagaje de estrategias didácticas sin ningún indicio novedoso, entre ellas: el dictado, las guías de estudios, las preguntas dirigidas, entre otras; ante las cuales el estudiantado suele reaccionar con poca motivación y expectativas hacia la clase y hacia el profesor, porque su rol es ser un "sujeto pasivo, reproductor del conocimiento" y el del profesorado es un sujeto "autoritario, rígido, controlador" (Rodríguez, 2013, p.43). También cabe agregar 
que una práctica tradicional se vuelve una monotonía perpetua en el salón de clase, y es entonces cuando el alumno se desmotiva, y el mismo docente también, porque se pierde el fin del aprendizaje y los indicadores de aprendizajes no son alcanzados satisfactoriamente.

Para ahondar en la discusión, en las prácticas tradicionales "se enseña a vivir a espaldas de la realidad, porque su esfuerzo se concentra en que los estudiantes, 'llenen su cabeza' de datos sencillos sin transferencia alguna en el entendimiento de lo que ocurre a su alrededor" (Santiago, 2008, p.16), es decir, el estudiante extrae poco provecho de los conocimientos que enseña la escuela ya que no los logra articular con su realidad, por ende, la enseñanza tradicional profundiza la desvinculación de los saberes escolares con las realidades sociales, prolongando así lo anunciado en el informe Delors (1996): aprendizajes para la vida.

En cuanto a la segunda, un modelo innovador parte del principio de que "es el resultado de una apuesta para mejorar un servicio, un producto o un recurso" (Gros y Lara, 2009, p.226), y también "se podría entender como el conjunto de ideas, procesos y estrategias (...) mediante las cuales se trata de introducir y provocar cambios en las prácticas educativas" (López, 2007, p.31). Por consiguiente, las prácticas innovadoras tienen la finalidad de presentar una nueva forma de alcanzar el aprendizaje, o bien, constituyen un conjunto de propuesta que presenta un medio distinto por el cual alcanzar los aprendizajes.

Este tipo de práctica docente se encuentran en las experiencias didácticas que presentan Pagés y Santisteban (2013), entre ellas: Profe... ipónganle lencería a la Historia! y Las salidas de campo: una estrategia dinamizadora para la enseñanza y el aprendizaje comprensivo de las Ciencias Sociales; asimismo, en el contexto educativo nicaragüense, las experiencias de Orozco y Díaz (2017) en La simulación como estrategia didáctica para desarrollar comprensión en la asignatura Historia. Intervención didáctica realizada en Educación Secundaria, y en Elaboración e interpretación de caricaturas como estrategia didáctica para el aprendizaje de la asignatura Filosofía en Educación Secundaria; demuestran que la innovación educativa se concretiza con la incorporación de cambios en los modelos pedagógicos existentes a fin de generar nuevos procesos de aprendizajes significativos y útiles para la vida.

No obstante, se habla de un modelo innovador cuando el profesorado, en primera instancia, logra percibir las necesidades de sus estudiantes, idear nuevas propuestas didácticas y llevar al aula nuevas experiencias didácticas que optimicen la calidad de los aprendizajes y que generen un aditivo motivacional en sus estudiantes. Es por tal razón que López (2007) plantea lo siguiente:

El principal problema en los procesos de carácter socioeducativo que pretenden ser innovadores, se centra en la búsqueda de la coherencia entre el discurso y la acción. 
La voluntad existe, la teoría se maneja, pero el no conocer "el cómo" llevarla a cabo, habitualmente consigue hacer naufragar las mejores intenciones y las iniciativas más innovadoras (p.32).

Lo aludido por López (2007) no está muy distante de las realidades educativas, algunos de los hallazgos encontrados en el estudio de Santiago (2008) indican que, aunque el profesorado cuenta con nuevos lineamientos didácticos para llevar a cabo experiencias novedosas en el aula de clase, en las prácticas educativas el tradicionalismo aún no logra superarse -algo semejante al contexto educativo nicaragüense-. Finalmente, el autor en mención destaca dos hallazgos de su estudio: la finalidad de la enseñanza de las ciencias sociales sigue siendo tradicional (el desarrollo de contenidos) y el dictado es una acción didáctica cotidiana (fomentando la memorización en el estudiantado).

Por consiguiente, las prácticas innovadoras siguen siendo un reto entre el profesorado. Todo indica que los discursos pomposos, plausibles y elocuentes, así como los escritos retóricos, convincentes y masificados, no han generado más que una impresión ambiciosa y utópica que no logra extrapolarse hacia las aulas de clase. Queda aún pendiente examinar la opinión del profesorado ante las reformas curriculares -posiblemente ese sea un factor que ayude a dilucidar este entramado-, pero lo que hoy es imprescindible eludir es el compromiso frontal que el profesorado debe asumir para incorporar más prácticas innovadoras en el aula de clase.

En este sentido, es por tal razón que el presente estudio consistió en conocer la opinión del estudiantado respecto a las metodologías innovadoras y tradicionales en la enseñanza de los estudios sociales en Educación Primaria, esto a partir de un proceso de intervención didáctica que se describirá en el transcurso del presente escrito.

\section{Metodología}

La presente investigación se llevó a cabo en el Colegio Público de Santo Domingo ubicado en la ciudad de Managua con estudiantes de Educación Primaria en la asignatura Estudios Sociales. El estudio consistió en una intervención didáctica que constó de diez sesiones de clase, y tuvo el objetivo de indagar la opinión que tienen los estudiantes de 5to grado de Educación Primaria respecto a las metodologías innovadoras y tradicionales que se utilizaron durante la intervención didáctica. Para ello, se realizaron 6 sesiones de clase con metodologías innovadoras y cuatro sesiones de clase con metodologías tradicionales, y finalmente se aplicó un cuestionario para recoger las percepciones de los estudiantes, tomando así el estudio una perspectiva cuantitativa, ya que la recolección de datos se fundamentó en la medición y se representaron mediante números (Hernández Sampieri, Fernández Collado y Baptista Lucio, 2014). 
Siguiendo a Hernández-Sampieri, Fernández-Collado y Baptista-Lucio (2014), el diseño en el cual se enmarca la presente investigación es experimental de tipo exploratorio, partiendo de que en un estudio experimental se "manipulan intencionalmente una o más variables independientes (...) para analizar las consecuencias que la manipulación tiene sobre una o más variables dependientes" (p.129), cabe aclarar que el término "manipular es sinónimo de hacer variar o asignar distintos valores a la variable independiente" (p.130); en este caso, se manipuló la variable estrategias didácticas, al implementar durante la primera fase de la investigación metodologías innovadoras y durante la segunda fase se implementaron metodologías tradicionales, a fin de conocer la opinión del estudiantado respecto a las mismas.

Así, la orientación exploratoria se refiere a que este tipo de estudio "en pocas ocasiones constituyen un fin en sí mismos. Generalmente (...) establecen el 'tono' de investigaciones posteriores más elaboradas y rigurosas" (p.91), es decir "sirven para familiarizarnos con fenómenos relativamente desconocidos, obtener información sobre la posibilidad de llevar a cabo una investigación más completa respecto de un contexto particular" (p.91), lo cual es algo que el presente estudio asumió, ya que pretende generar un espacio de reflexión entre el profesorado con el objetivo de motivar al profesorado a realizar experimentos didácticos en el aula de clase.

\section{Resultados}

\section{Descripción de la intervención didáctica}

La intervención didáctica se llevó a cabo con estudiantes de 5to grado de Educación Primaria en la asignatura Estudios Sociales a través de diez sesiones de clase en las cuales se abordaron dos contenidos: El poblamiento de América, impartido con metodologías innovadoras durante seis sesiones de clase, y el contenido El descubrimiento de América, impartido con metodologías tradicionales durante cuatro sesiones de clase, esto con el fin de alcanzar los objetivos propuestos, es decir, conocer la opinión del estudiantado en cuanto a las metodologías tradicionales e innovadoras.

A continuación, se hará una escueta descripción de cada sesión de aprendizaje desde la perspectiva del docente investigador, destacando las estrategias didácticas utilizadas, el ambiente de aprendizaje y los resultados obtenidos en las mismas:

La sesión didáctica no.1 inició con una dinámica de presentación para generar empatía entre docente y estudiantes. El objetivo de esta sesión didáctica consistió en explorar los conocimientos previos que los estudiantes tenían acerca de los contenidos El poblamiento de América y El descubrimiento de América, para ello se implementó una prueba diagnóstica basado en el instrumento SQA, la cual fue resuelta individualmente, luego socializada y discutida en grupo, y finalmente valorada como novedosa por el estudiantado. 


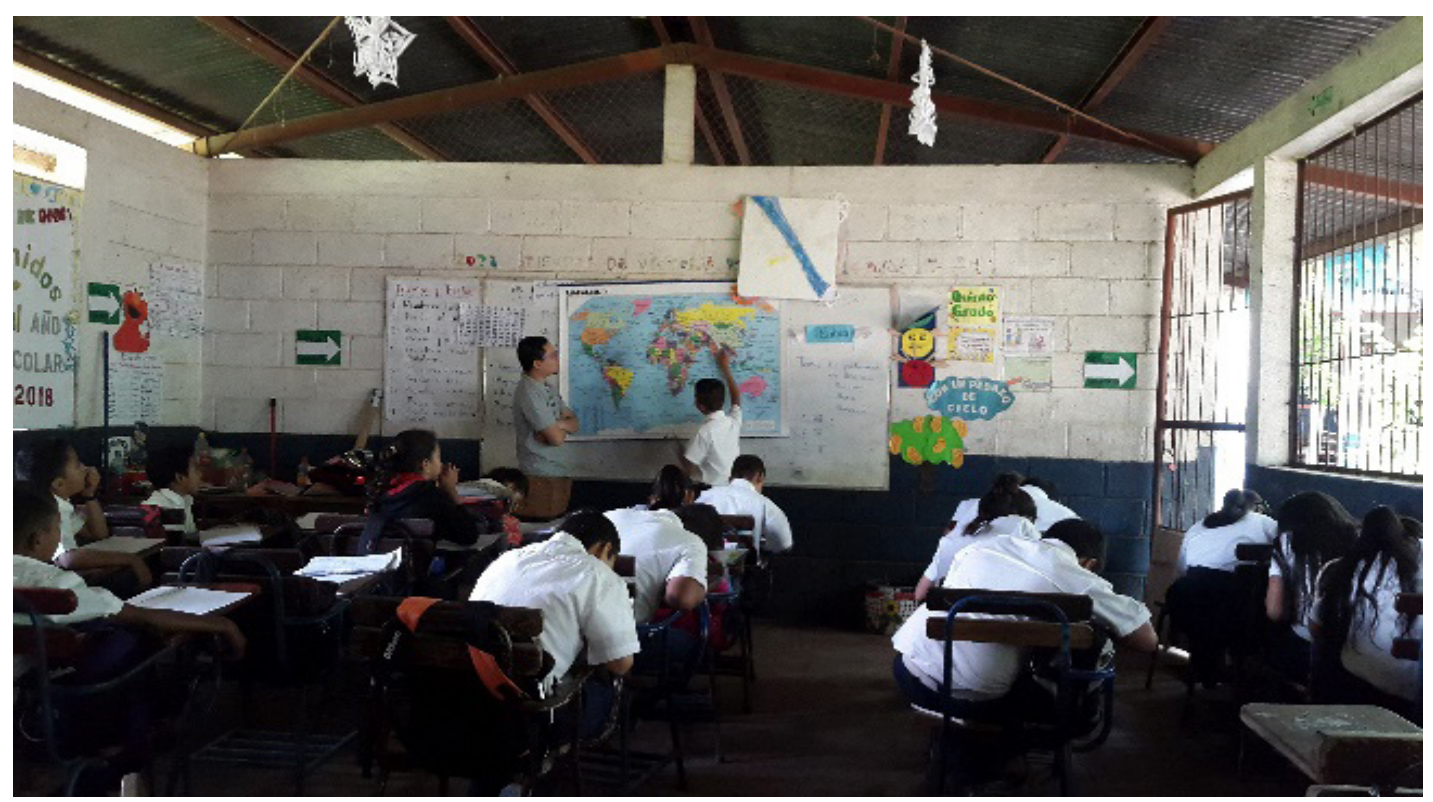

Figura 1. Estudiante explicando las teorías del poblamiento de América.

En la sesión didáctica no.2 se impartió el sub-contenido Las teorías del poblamiento de América, en donde el docente utilizó un mapamundi para explicar las tres teorías del poblamiento de América y, por otra parte, el estudiantado debía realizar esquemas en donde reflejaran las características principales de cada teoría y posteriormente participar en el conversatorio en clase. La sesión didáctica no.3 fue práctica. Inició con una recapitulación de las tres teorías del poblamiento de América dirigida voluntariamente por un estudiante. El desarrollo de la clase se dio a través de una narrativa-expositiva y con el apoyo del mapamundi, el docente profundizó en la explicación de las teorías del poblamiento de América y paralelamente el estudiantado debía ir graficando y explicando en un mapamundi cada una de ellas. Esta actividad fue muy positiva para el estudiantado, quienes manifestaron que era una actividad novedosa y práctica porque coloreando y dibujando podían representar sus aprendizajes.

En cuanto a la sesión didáctica no.4, se abordó el sub-contenido Ubicación geográfica de los primeros pobladores de América y Centroamérica mediante una narrativa, en donde el profesor, con apoyo de un mapa de América, iba describiendo la ubicación geográfica de los primeros pobladores, y el estudiantado iba ubicándolos en un mapa de América. Esta actividad fue muy práctica y grata para el estudiantado, y les permitió conocer el área geográfica de las distintas tribus indígenas que habitaban América previo a la colonización. 


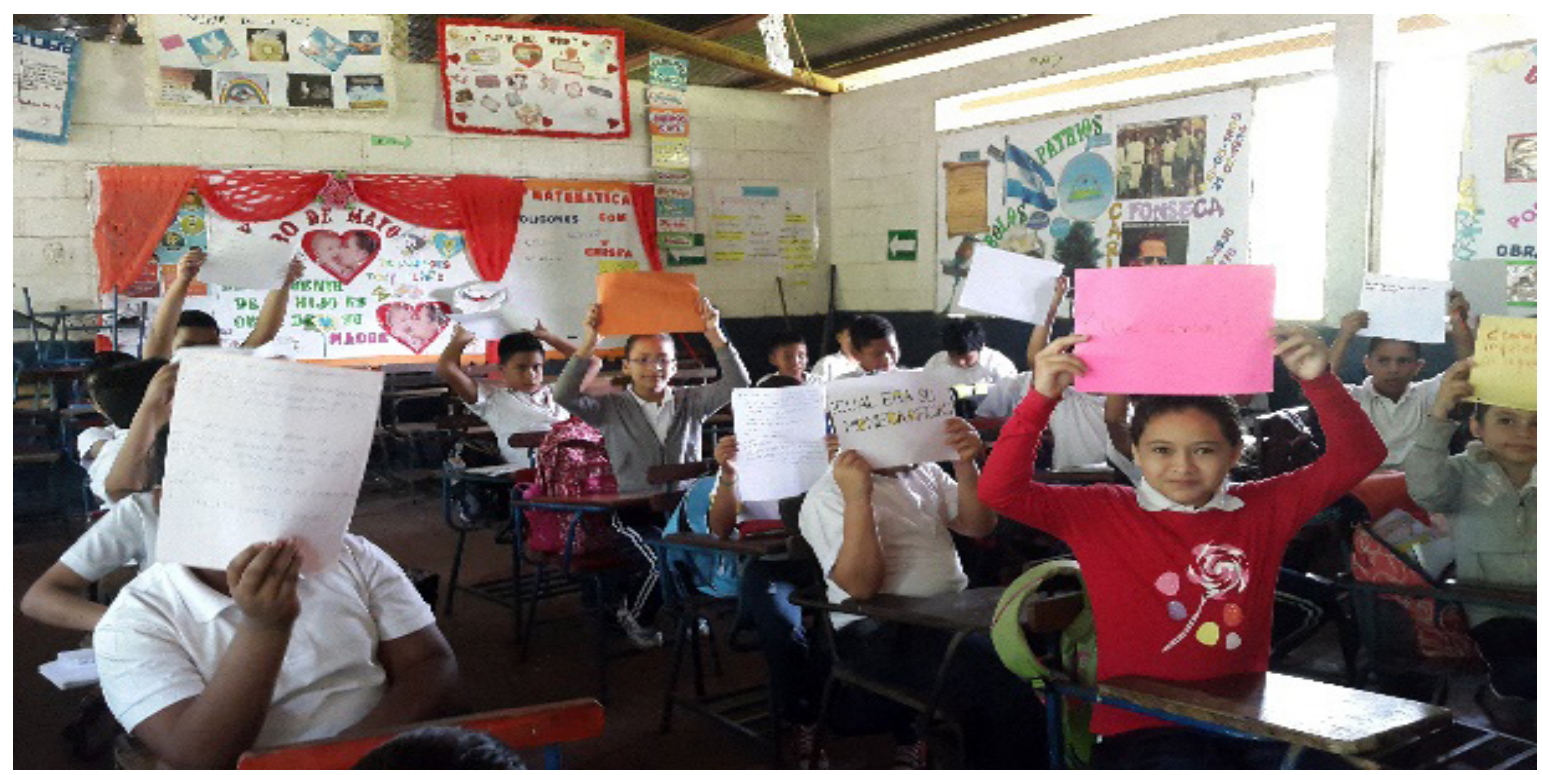

Figura 2. Estudiantes presentando sus curiosidades sobre las actividades económicas de los primeros pobladores.

En la sesión didáctica no. 5 se abordó el sub-contenido Actividades económicas y culturales de los primeros pobladores a través de la estrategia didáctica elaboración de curiosidades. La actividad consistía que el estudiantado debía escribir en una hoja una curiosidad respecto a las actividades económicas y culturales que realizaban los primeros pobladores, y el docente, mediante un conversatorio participativo, daba respuestas mientras los estudiantes tomaban notas. Algunas de las curiosidades que anotaron los estudiantes fueron las siguientes: ¿Cuál era su moneda oficial? ¿Cómo se divertían los indígenas? ¿Cómo hacían su comida? ¿En qué trabajaban las mujeres? ¿Cuál era el mecanismo de educación de los indígenas?

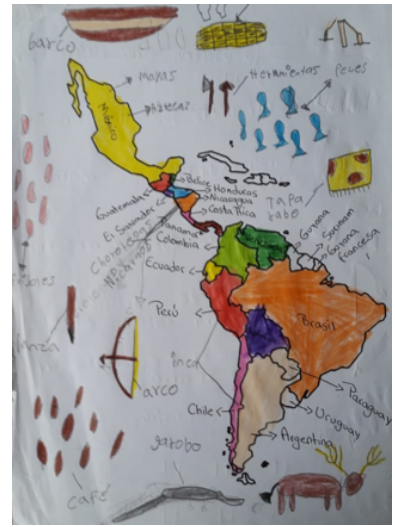

Figura 3. Mapa sobre las actividades económicas de los aborígenes
En el caso de la sesión didáctica no.6, su finalidad fue que los estudiantes representaran en un mapa de América las actividades económicas de los primeros pobladores del continente. Para ello, la clase inició con una simulación, un estudiante ilustró uno de los instrumentos que los antepasados utilizaban para realizar actividades económicas como la caza. Luego el docente en conjunto con los estudiantes realizó una recapitulación de la clase pasada y paralelamente los estudiantes debían ir dibujando las distintas actividades económicas que practicaban los aborígenes. Y en el segundo momento de la clase, los 
estudiantes elaboraron una caricatura sobre tales actividades, las cuales evidenciaron sus comprensiones. Cabe destacar que ambas actividades resultaron ser novedosas para los estudiantes, inclusive, muchos de ellos desconocían qué era una caricatura.

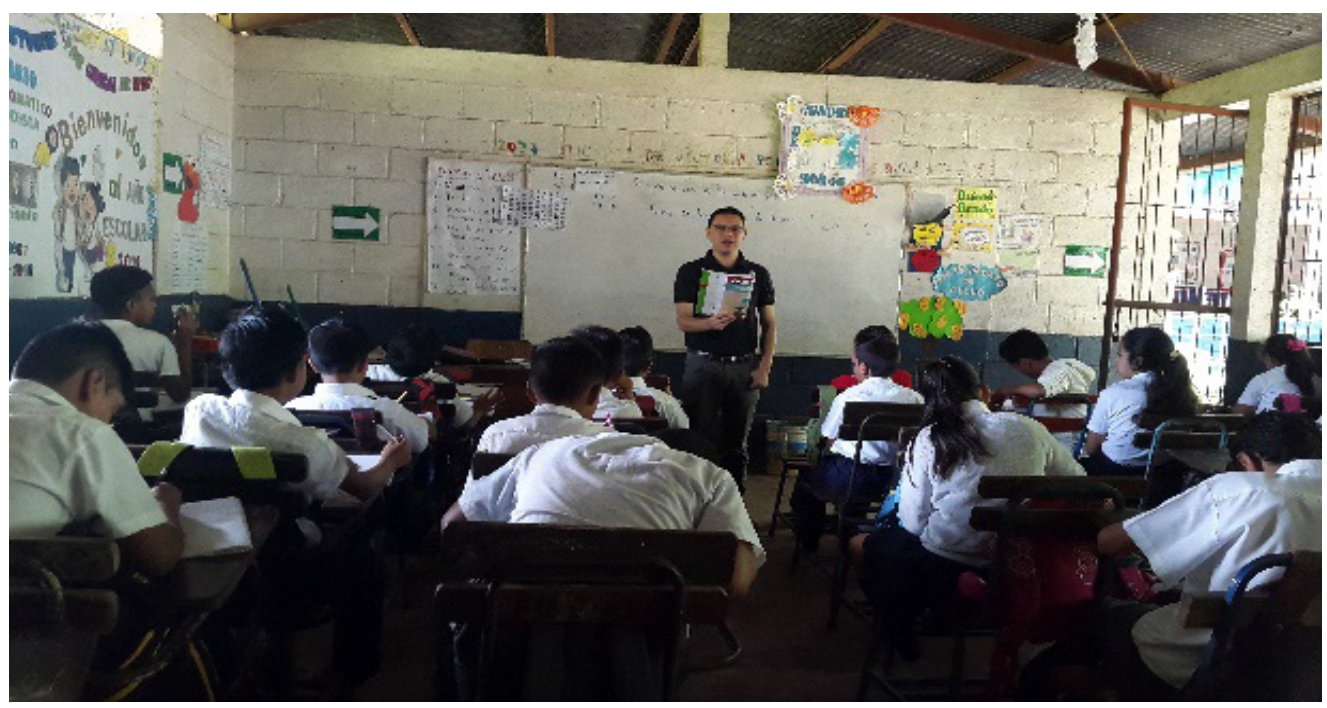

Figura 4. Profesor dictando el contenido causas del descubrimiento de América

Por otra parte, con la sesión didáctica no.7 se inicia la fase de la aplicación de metodologías didácticas tradicionales, y se inició aplicando la estrategia didáctica del dictado con el sub-contenido Causas del descubrimiento de América. La sesión didáctica se desarrolló en silencio y sin participación del estudiantado, el maestro dictó únicamente, copió en el pizarrón algunas palabras que los estudiantes desconocían cómo se escribía y, por otra parte, los estudiantes únicamente se dedicaron a copiar el contenido.

Asimismo, en la sesión didáctica no.8 la estrategia didáctica que se implementó fue el cuestionario. El profesor inició dictando alguna información para complementar el tema anterior, y posteriormente dictó un cuestionario con preguntas conceptuales a los estudiantes, quienes debían contestarlas en grupo y posteriormente entregarlas al profesor para que éste las calificase. Se observó que no todos los integrantes de los grupos realizaban la actividad, algunos de ellos esperaban que su compañero encontrara las respuestas para posteriormente copiarlas en su cuaderno. 


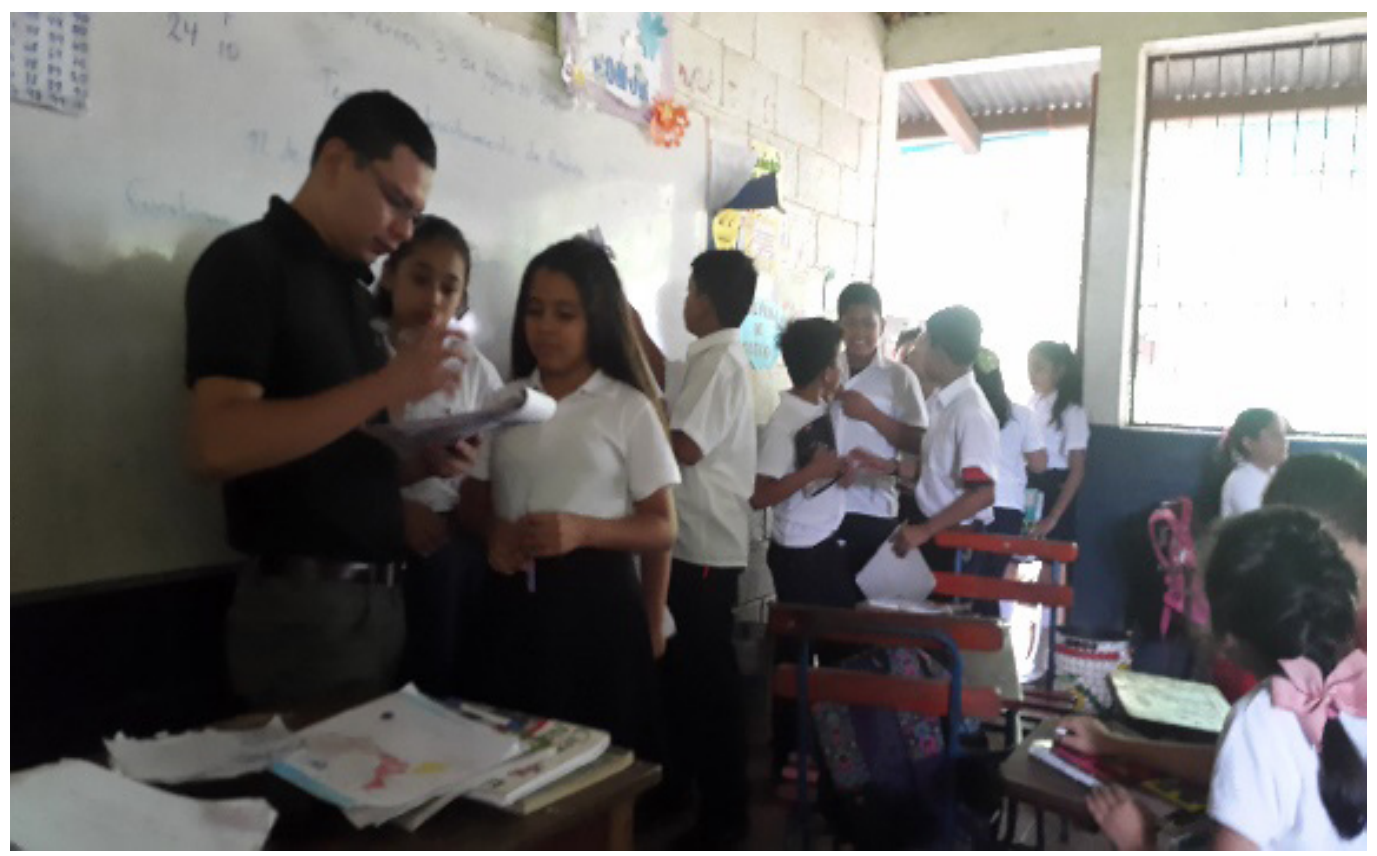

Figura 5. Profesor revisando cuestionario a los estudiantes

En el caso de la sesión didáctica no.9 se impartió el contenido Consecuencias del descubrimiento de América, y este se desarrolló de la siguiente manera: se les facilitó un texto a los estudiantes, y ellos debían de copiarlo en su cuaderno. Posteriormente el profesor dictó un cuestionario literal que los estudiantes debían resolver para luego ser entregado y calificado por el docente. En esta clase se observó que el estudiantado trabajaba en grupo con mucha prisa mientras el docente se dedicaba a observarlos únicamente.La sesión didáctica no.1o fue de evaluación. El profesor asumió un rol tradicional al aplicar los instrumentos de investigación: formó a los estudiantes en hileras iguales, distribuyó las evaluaciones, se sentó a observar a los estudiantes, recibió las evaluaciones ya resueltas, y finalmente se retiró del salón, calificó las evaluaciones y al siguiente día únicamente las entregó, es decir, no hubo socialización de las mismas, solamente se cumplió con la actividad sin poner atención a las dificultades que habían tenido los estudiantes en las evaluaciones, y sin hacer una recapitulación de las mismas para fortalecer los saberes.

\section{Opinión del estudiantado sobre las metodologías innovadoras y tradicionales.}

Una vez culminada la fase de la intervención didáctica, el profesor investigador distribuyó un cuestionario a los estudiantes para que evaluasen las metodologías implementadas durante la intervención didáctica, el mismo estuvo enfocado específicamente en comparar ambas metodologías a fin de obtener la opinión del estudiantado. A continuación, se presentan los resultados del cuestionario: 
1. Los estudiantes prefieren el contenido que se impartió con la metodología tradicional. El primer ítem del cuestionario indica que al 69\% de los estudiantes les gustó más el contenido El descubrimiento de América que fue impartido con metodologías tradicionales. A diferencia del contenido El poblamiento de América, en donde solamente un 31\% expresó que les gustó más la metodología didáctica con que se impartió el contenido en mención. Esto deja entrever que el estudiantado lleva consigo una enorme carga del modelo tradicional en su forma de concebir los procesos de aprendizajes.

2. Los estudiantes prefieren las actividades de aprendizajes desarrolladas con metodologías innovadoras. Las actividades de aprendizajes se conciben como el conjunto de acciones que el estudiantado realiza para alcanzar los fines de aprendizajes propuestos, en este sentido, se les pidió que expresaran cuáles de las actividades realizadas les había gustado más, los resultados indicaron que los estudiantes prefieren las actividades innovadoras sobre las tradicionales. En la Figura 6 se muestran los resultados:

a. Elaborar mapa del poblamiento de América

b. Dibujar las actividades económicas de los primeros pobladores

c. Conversar sobre curiosidades de nuestros primeros pobladores

d. Elaborar una caricatura

e. Copiar del dictado del descubrimiento de América

f. Elaborar esquemas en el cuaderno

g. Copiar del libro de texto sobre el descubrimiento de América

h. Contestar preguntas sobre el descubrimiento de América

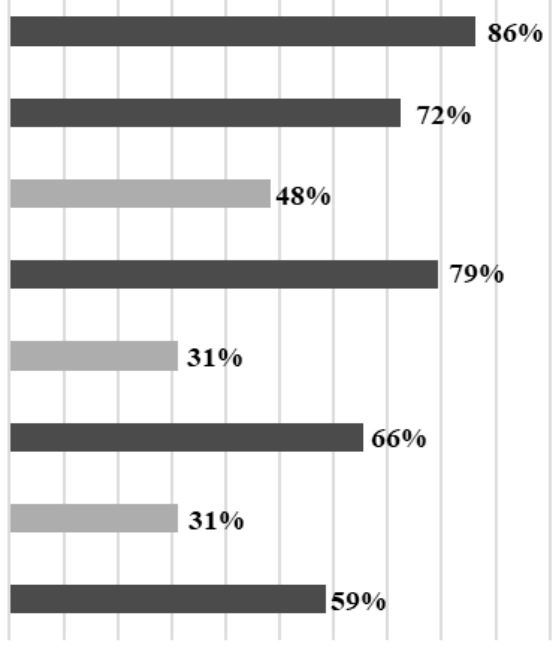

Figura 6. Actividades de aprendizajes que más les gustaron a los estudiantes

3. Los estudiantes prefieren que el docente asuma un rol innovador al momento de impartir la clase. El rol del profesorado en el aula de clase es determinante para definir qué tipo de aprendizaje se va a generar en los estudiantes, ya que a través de este se le puede asignar un rol activo o pasivo al estudiantado. Los resultados indicaron que el estudiantado prefiere a docentes activos que generen interacción en su clase a través preguntas directas, narraciones interactivas y conversatorios, no así con las metodologías tradicionales como copiar en la pizarra, dictar y revisar las tareas por hilera como la escuela tradicional suele practicarlo. 


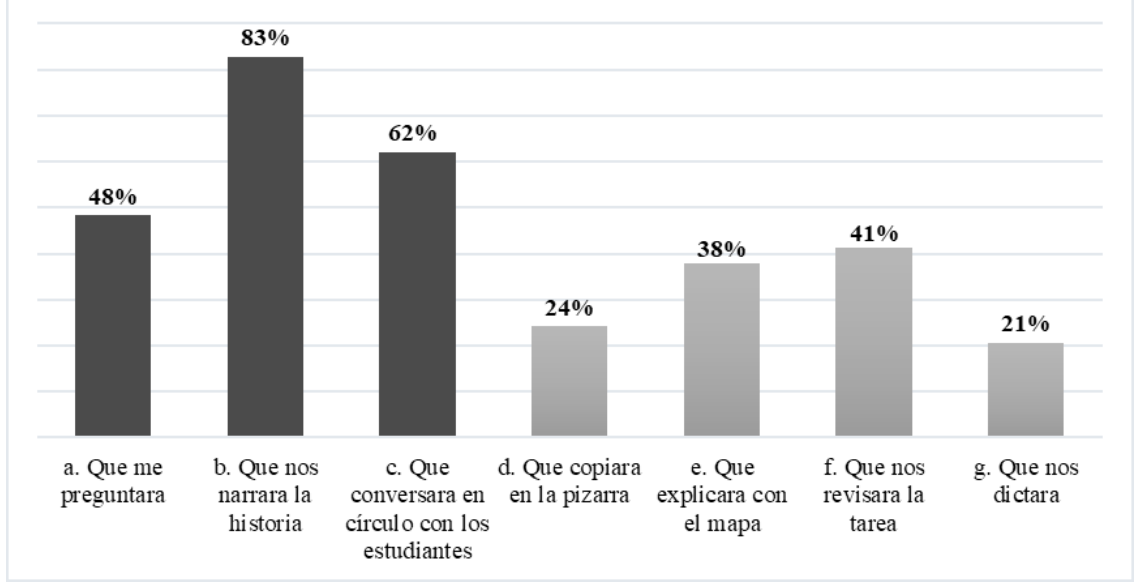

Figura 7. Valoración del rol del profesor en el aula de clase

4. Los estudiantes prefieren las evaluaciones tradicionales. Para evaluar los aprendizajes que los estudiantes habían adquirido de ambos contenidos impartidos se aplicaron dos instrumentos de evaluación: El contenido El poblamiento de América, impartido con metodologías innovadoras, se evaluó con un instrumento innovador en donde los estudiantes debían graficar, dibujar y analizar una caricatura; y el contenido El descubrimiento de América, impartido a través de metodologías tradicionales se evaluó con un instrumento tradicional que constaba de ítemes de Falso y Verdadero, selección múltiple y preguntas de desarrollo. Al respecto, la opinión del estudiantado fue la siguiente: El 66\% prefirió la prueba tradicional y solo el $34 \%$ optó por la prueba innovadora; lo cual indica la tendencia que tiene el estudiantado que ha sido formado bajo el paradigma tradicional.

5. Los estudiantes prefieren aprender con metodologías innovadoras.

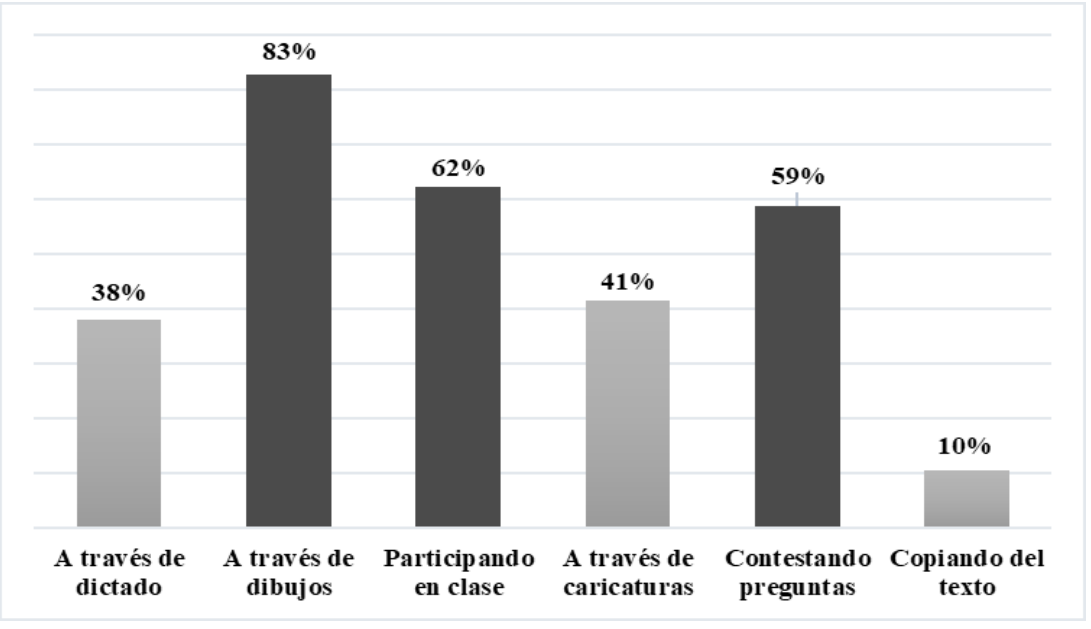

Figura 8. Metodologías con las cuales los estudiantes prefieren aprender Estudios Sociales 
Finalmente, se le preguntó al estudiantado ¿De qué manera te gusta más aprender Estudios Sociales?, a lo cual el estudiantado con abrumadora ventaja optó por las metodologías innovadoras, entre ellos la utilización de dibujos, clases participativas e interactivas. Sin embargó, los resultados también demostraron que otra parte del estudiantado también opta por metodologías tradicionales como los cuestionarios conceptuales y el uso del dictado.

\section{Discusión y conclusiones}

\section{El camino hacia la innovación es un imperativo}

Los resultados hasta acá expuesto apuntan a algo en común: existe una lucha entre la permanencia de metodologías tradicionales y la incorporación paulatina de metodologías innovadoras. El estudiantado -pese a contener una enorme carga tradicional- tiene una clara tendencia hacia lo innovador -y no es de asombrar-, ya que la sociedad actual pone a disposición de ellos un conjunto de recursos tecnológicos con los cuales cohabita permanentemente, y estos exigen establecer otro tipo de relación con su medio, por consiguiente, con la escuela que es el espacio geográfico en donde el estudiantado reside gran parte de su tiempo.

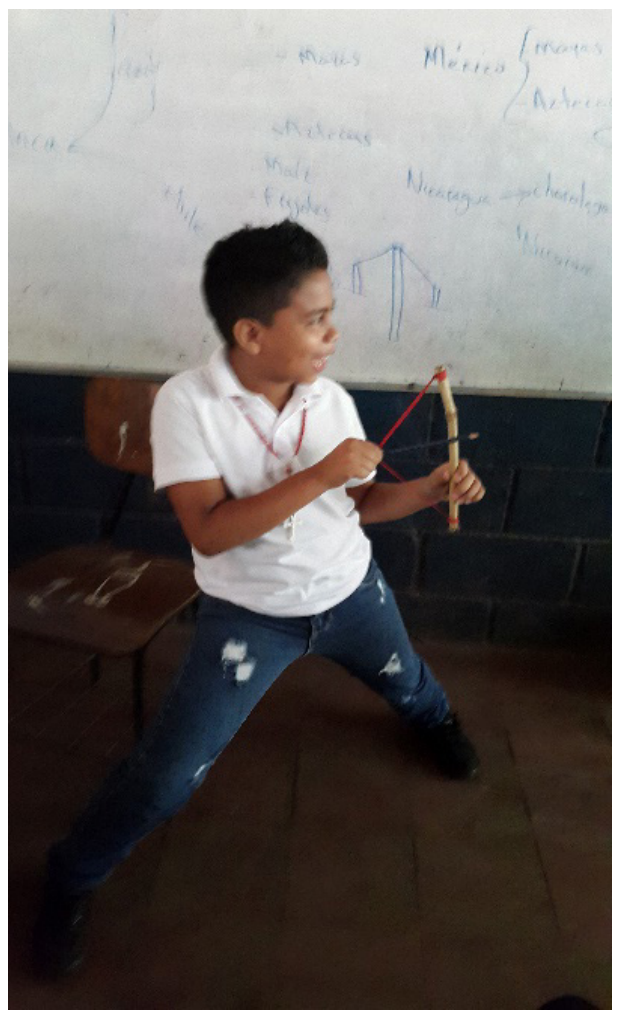

Figura 9. Estudiante realiza simulación de instrumento de caza. 
En definitiva, los retos para el profesorado en la sociedad actual son crecientes. El profesor de hoy ha de llevar a cabo una lucha incesante entre lo tradicional y lo innovador para evitar apelativos desdeñosos como: la clase es aburrida, la clase es tradicional, el profesor enseña a la antigua, esa clase me da sueño, entre tantas otras expresiones que el estudiantado puede interpelar ante la desmotivación hacia la metodología del profesor y, por ende, hacia la asignatura que imparte tal profesor. No obstante, el profesorado debe asumir el enorme reto de la innovación: reflexionar sobre su práctica educativa, incorporar mejoras en los procesos de aprendizajes que dirige, adoptar nuevas metodologías didácticas e insertar en sus metodologías didácticas elementos novedosos que logren despertar la curiosidad y la creatividad en el estudiantado, porque "para que la innovación educativa sea real y efectiva, la labor del profesorado se configura en una pieza vita del engranaje" (López, 2007, p.32).Finalmente decir que, el presente estudio fue diseñado desde una perspectiva metodológica exploratoria con el fin de suministrar datos concretos sobre la percepción que tiene el estudiantado sobre las metodologías innovadoras y participativas, dejando abierta deliberadamente una enorme ventana para futuras investigaciones exploratorias y experimentales, para así entrar en este exuberante campo de estudio y dar respuestas a las demandas de los estudiantes, a través de la promoción de metodologías innovadoras en la enseñanza de las ciencias sociales, y la formación de profesores de ciencias sociales que afronten oportunamente las demandas que el contexto educativo exige: El camino hacia la innovación es un imperativo.

\section{Lista de referencias}

Delors, J. et al (1996). La Educación Encierra un Tesoro. Unesco: Santillana.

Gros, B. y Lara, P. (2009). Estrategias de Innovación en la Educación Superior: El caso de la Universitat Oberta en Catalunya. Revista Iberoamericana de Educación, (49): 223-245.

Hernández Sampieri, R., Fernández Collado, C., y Baptista Lucio, M. (2014). Metodología de la Investigación. (6ta edición). México: McGRAW-HILL.

López, F. (2007). Metodología participativa en la Enseñanza Universitaria. España: NARCEA.

Orozco, J. y Díaz, A. (2017, marzo). La simulación como estrategia didáctica para desarrollar comprensión en la asignatura Historia. Intervención didáctica realizada en Educación Secundaria. Revista Científica de FAREM-Estelí. Medio ambiente, tecnología y desarrollo humano, (6):4-13. Recuperado el 25 de septiembre de 2018, de https://www.lamjol.info/index.php/FAREM/article/view/3481/3234 
Orozco, J. y Díaz, A. (2017, mayo). Elaboración e interpretación de caricaturas como estrategia didáctica para el aprendizaje de la asignatura Filosofía en Educación Secundaria. Torreón Universitario, (15):6-24. Recuperado el 25 de septiembre de 2018, de https://www.lamjol.info/index.php/torreon/article/view/5559/5257

Pagés, J. y Santisteban, A. (2013). Una mirada del pasado al futuro en la didáctica de las ciencias sociales. (Vol. 1). Barcelona: Asociación Universitaria de Profesorado de Didáctica de las Ciencias Sociales.

Rodríguez, J. (2013, junio). Una mirada a la pedagogía tradicional y humanista. Presencia Universitaria, (5):36-45. Recuperado el 21 de septiembre de 2018, de http:// eprints.uanl.mx/3681/1/Una_mirada_a_la_pedagog\%C_\%ADa_tradicional_y_ humanista.pdf

Santiago, J. (2008). La enseñanza de las ciencias sociales en la escuela bolivariana. Geoenseñanza, 13(1):5-18. Recuperado el 21 de septiembre de 2018, de https:// www.redalyc.org/pdf/360/36014579002.pdf 